\title{
CRITICAL EPIDEMICS, RANDOM GRAPHS, AND BROWNIAN MOTION WITH A PARABOLIC DRIFT
}

\author{
REMCO VAN DER HOFSTAD, ${ }^{* * *}$ Eindhoven University of Technology \\ A. J. E. M. JANSSEN, ${ }^{* * *}$ EURANDOM and Eindhoven University of Technology \\ JOHAN S. H. VAN LEEUWAARDEN, ${ }^{* * * * *}$ Eindhoven University of Technology
}

\begin{abstract}
We investigate the final size distribution of the SIR (susceptible-infected-recovered) epidemic model in the critical regime. Using the integral representation of Martin-Löf (1998) for the hitting time of a Brownian motion with parabolic drift, we derive asymptotic expressions for the final size distribution that capture the effect of the initial number of infectives and the closeness of the reproduction number to zero. These asymptotics shed light on the bimodularity of the limiting density of the final size observed in Martin-Löf (1998). We also discuss the connection to the largest component in the Erdôs-Rényi random graph, and, using this connection, find an integral expression of the Laplace transform of the normalized Brownian excursion area in terms of Airy functions.
\end{abstract}

Keywords: SIR epidemic model; final size distribution; critical random graph; largest connected component; phase transition; Airy function; Brownian motion with parabolic drift

2010 Mathematics Subject Classification: Primary 60C05; 05C80; 90B15

\section{Introduction}

Amongst the most popular mathematical models for the spread of a contagious disease is the susceptible-infected-recovered (SIR) epidemic model, in which all individuals of a finite population interact in the same manner. Individuals are either susceptible, infected, or recovered, and at time $t$ the population state can be defined by the vector $\left(S_{t}, I_{t}\right)$, where $S_{t}$ and $I_{t}$ denote the numbers of susceptible and infected individuals, respectively. In the simplest SIR model, also known as the Reed-Frost model, individuals remain infected for one unit of time, after which they recover and acquire permanent immunity from future infection, and, hence, $I_{t+1}=S_{t}-S_{t+1}, t \in \mathbb{N}$. During any period $(t, t+1)$, the disease is transmitted from $i \in I_{t}$ to $s \in S_{t}$ with probability $p$, and all encounters per time unit between any pairs of individuals are assumed to be independent of each other. Therefore, given $\left(S_{t}, I_{t}\right)$ and $q=1-p, S_{t+1}$ follows a binomial distribution:

$$
S_{t+1} \stackrel{\mathrm{D}}{=} \operatorname{Bin}\left(S_{t}, q^{I_{t}}\right), \quad t \in \mathbb{N},
$$

where $\stackrel{\text { D }}{=}$ denotes equality in distribution. That is, during any period $(t, t+1)$, each infected individual fails to transmit the disease to any given susceptible individual with probability $q$.

\footnotetext{
Received 17 May 2010.

* Postal address: Department of Mathematics and Computer Science, Eindhoven University of Technology, PO Box 513, 5600 MB Eindhoven, The Netherlands.

** Email address: rhofstad@win.tue.nl

*** Postal address: Department of Electrical Engineering, Eindhoven University of Technology, PO Box 513, 5600

MB Eindhoven, The Netherlands. Email address: a.j.e.m.janssen@tue.nl

**** Email address: j.s.h.v.leeuwaarden@tue.nl
} 
Each individual at time $t$ was exposed to a possible infectious encounter with any of the infected individuals, and each susceptible individual has a probability of $q^{I_{t}}$ of escaping the disease in that time slot. The epidemic will die out the first time, $T$, there are no more infected individuals, so $T=\inf \left\{t: I_{t}=0\right\}$, and the final size of the epidemic is then $N-S_{T}=I_{1}+\cdots+I_{T-1}$.

The final size of the epidemic will strongly depend on the initial conditions $\left(S_{0}, I_{0}\right)$ and the infection parameter $p$. The final size in the simple SIR model and its many extensions has been a popular object of study, and it has long been known that the final size distribution in many cases is bimodal (two local maxima). This bimodal feature is caused by two likely scenarios: either the epidemic dies out quickly, infecting few individuals, or the epidemic becomes long and substantial. The likeliness of the scenarios depends crucially on the socalled reproduction number $\lambda_{n}=n p$. When studying the epidemic in large populations, $\lambda_{n}$ is close to the mean offspring of a branching process, and, hence, when $\lambda_{n}<1$, the epidemic will end quickly, even if $I_{0}$ is large, while if $\lambda_{n}>1$, there is a positive probability (roughly 1 minus the extinction probability in the branching process) that the epidemic will be large. The large- $n$ behavior in both cases is well understood [14], and shows great resemblance to the behavior of branching processes. The epidemic becomes more interesting when $\lambda_{n} \approx 1$, in which case rather surprising large- $n$ limit results can be obtained. The most pronounced case occurs when $\lambda_{n}$ is just slightly larger than 1. For a general class of SIR models, Martin-Löf [14] derived the so-called threshold limit theorem for the asymptotic regime when the population size is large. Indeed, under the appropriate normalization, the process of infectives weakly converges to a scaling limit when, simultaneously, the total population $n$ approaches $\infty$, while $\lambda_{n}$ approaches 1 .

Theorem 1.1. (The critical behavior [2], [15].) Assume that $p=p_{n}=\lambda_{n} / n$, let

$$
a=\lim _{n \rightarrow \infty} n^{1 / 3}\left(\lambda_{n}-1\right),
$$

and assume that the number $I_{0}^{n}$ of initially infected individuals is such that

$$
\lim _{n \rightarrow \infty} \frac{I_{0}^{n}}{n^{1 / 3}}=b .
$$

Then, as the population size $n \rightarrow \infty$, the final size of the epidemic $K_{n}$ has the limit distribution

$$
K_{n} / n^{2 / 3} \stackrel{\mathrm{D}}{\rightarrow} T^{a}(b),
$$

where $T^{a}(b)$ is the first passage time to the level $-b$ by $\left(W_{t}+a t-t^{2} / 2\right)_{t \geq 0},\left(W_{t}\right)_{t \geq 0}$ is a standard Wiener process, and $\stackrel{\mathrm{D}}{\rightarrow}$ ' denotes convergence in distribution.

See also [2] and [18]. There are several important observations from Theorem 1.1. When the number of initially infected individuals $I_{0}$ is sufficiently small, relative to the population size, the epidemic evolves approximately as a Galton-Watson process with Poisson(1) offspring distribution. However, when $I_{0}$ passes some critical threshold, the epidemic will behave quite differently from the branching process. This critical threshold can be seen from Theorem 1.1 to be $n^{1 / 3}$. Next, the critical window turns out to be

$$
p_{n}=\frac{1}{n}\left(1+\frac{a}{n^{1 / 3}}\right), \quad a \in \mathbb{R},
$$

and the final epidemic size is of order $n^{2 / 3}$.

Martin-Löf [15] used methods from Groeneboom [8] to derive an exact solution for the density of the first passage time $T^{a}(b)$. Let $\operatorname{Ai}(x)$ and $\operatorname{Bi}(x)$ denote the classical Airy functions (see [1]). 
Theorem 1.2. (The first passage time density [15].) The first passage time $T_{\sigma}^{a}(b)$ of $\sigma W_{t}$ to the parabola $b+a t-t^{2} / 2$ has probability density

$$
f_{b}(t ; a, \sigma)=\mathrm{e}^{-\left((t-a)^{3}+a^{3}\right) / 6 \sigma^{2}-a x} \int_{-\infty}^{\infty} \mathrm{e}^{t u} \frac{B(u) A(u-x)-A(u) B(u-x)}{\pi\left(A^{2}(u)+B^{2}(u)\right)} \mathrm{d} u,
$$

where $A(u)=\operatorname{Ai}(c u), B(u)=\operatorname{Bi}(c u), c=\left(2 \sigma^{2}\right)^{1 / 3}$, and $x=b \sigma^{-2}>0$.

Note that $T^{a}(b)=T_{1}^{a}(b)$. The integral expression in (1.1) can be employed to compute the density numerically (although difficulties arise for small $t$ ). This is useful because computing the distribution of $K_{n}$ for large values of $n$ is computationally cumbersome, which is why recursive algorithms have been developed (see [7] and the references therein). In [7] such algorithms have been used to calculate the pre-limit density and to compare it against the limiting density in (1.1). It was shown that the rate of convergence is rather fast, so that the limit result yields good approximations for the finite population, provided $n$ is large enough. Hence, for a large fixed population size $n$, with reproduction number $\lambda_{n}$ and initial number of infectives $m$, estimates of $a$ and $b$ follow from $\lambda_{n} \approx 1+a n^{-1 / 3}$ and $m \approx b n^{1 / 3}$, and the final size $K_{n}$ is well approximated by $T^{a}(b) n^{2 / 3}$.

Our goal in this paper is to reveal the structural properties of the density in (1.1) in order to obtain detailed information on how the critical epidemic evolves as a function of the initial number of infectives and the closeness of the reproduction number to zero. We will employ the integral in (1.1) to derive much simpler asymptotic expressions for the density around zero, the tail probabilities, and also for small and large $a$ and $b$.

When $a$ and $b$ are large enough, the second maximum is the global maximum and occurs at roughly $t=a+\sqrt{a^{2}+b}$, which is the point where the parabola crosses the $t$-axis. Regarding the bimodality, the following observations were made in [15] based on numerical investigations. There is a critical $b^{*}$ such that, if $b>b^{*}$, there is only one maximum for all values of $a$. When $b<b^{*}$, there is a critical $a^{*}$ (that depends on $b$ ) such that the density is unimodal if $a<a^{*}$ and bimodal if $a>a^{*}$. Hence, for the density to have two local maxima, $b$ should be small enough, while $a$ should be large enough. See Figure 1 for an example.

To further illustrate the influence of $a$ and $b$, we show in Figure 2 a sample path of the Brownian motion and the parabola $b+a t-t^{2} / 2$ with $a=b=1$. In this example $T^{a}(b) \approx 2.44$. It is clear that on average the hitting time $T^{a}(b)$ increases with $a$ and $b$. The hitting time can be

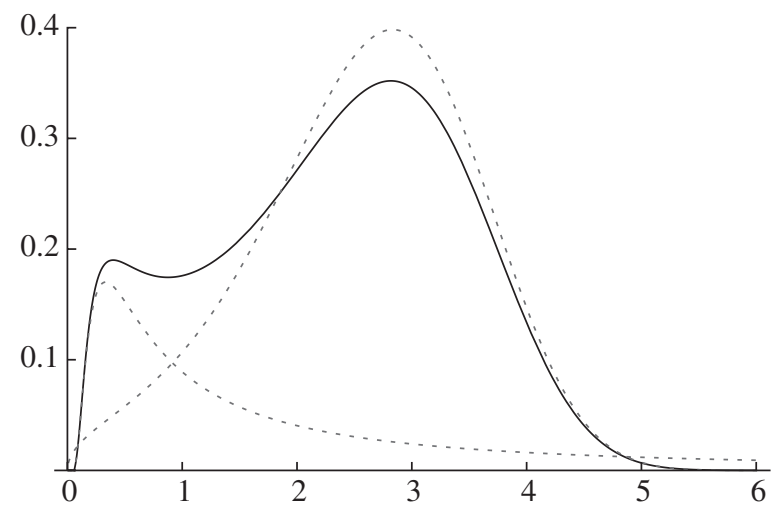

FIGURE 1: Density $f_{b}(t ; a, \sigma)$ (solid line) and the approximations in (2.3) and (2.4) (dashed lines) for $a=b=\sigma=1$. 


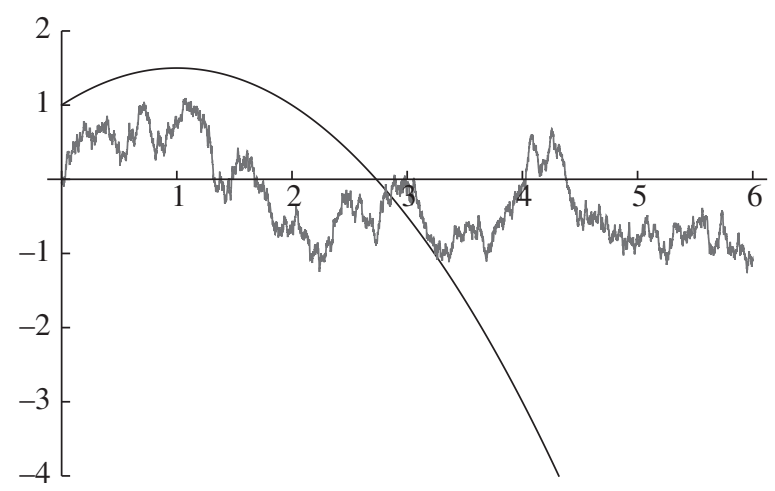

Figure 2: Wiener process and the parabola $1+t-t^{2} / 2$.

interpreted as a competition between the parabola and the Brownian motion, where $b$ represents the headstart given to the parabola, and $a$ gives that parabola even more edge in the initial stages. This fits with our intuition that a larger initial number of infectives and a larger reproduction number result in a larger epidemic. For the epidemic to die out quickly, despite the headstart $b$ and the initial drift $a$, requires a highly unlikely trajectory of the Brownian motion. This is quantified in Theorem 2.3.

We present our main results in Section 2 concerning new asymptotic expressions for the final size distribution. SIR models have much in common with random graphs (see [12]). In particular, the Erdős-Rényi random graph pertains to the same critical regime as in Theorem 1.1. In Section 3 we further describe this intimate connection between the final size in the SIR model and the largest connected component in random graphs. In Section 4 we discuss how our results for the final size distribution carry over to much larger classes of SIR models and random graphs. The proofs of our main results are presented in Sections 5 and 6.

\section{Main results}

We see from (1.1) that the first passage time density can be written as

$$
f_{b}(t ; a, \sigma)=\mathrm{e}^{-\left((t-a)^{3}+a^{3}\right) / 6 \sigma^{2}-a x} \frac{1}{c} I\left(\frac{t}{c} ; x c\right)
$$

with

$$
I(t, x)=\int_{-\infty}^{\infty} \mathrm{e}^{t u} \frac{\operatorname{Bi}(u) \operatorname{Ai}(u-x)-\operatorname{Ai}(u) \operatorname{Bi}(u-x)}{\pi\left(\operatorname{Ai}^{2}(u)+\operatorname{Bi}^{2}(u)\right)} \mathrm{d} u .
$$

In this section we present several asymptotic results for the first passage time density. The proofs, which are presented in Sections 5 and 6, rely on various approximations for the Airy functions and techniques for the asymptotic evaluation of integrals. In fact, the main task consists of analyzing in full detail the integral $I(t, x)$ for $t>0$ and $x>0$.

We have the following results.

Theorem 2.1. Uniformly in $x \in[0, D]$, where $D>0$ is fixed,

$$
I(t, x)=\frac{x}{2 \pi t}\left(\frac{\pi}{t}\right)^{1 / 2} \mathrm{e}^{-x^{2} / 4 t}\left(1+\frac{1}{2} x t\right)+O(1), \quad t>0 .
$$


Theorem 2.2. Uniformly in $x \in[\varepsilon, D]$ for any $\varepsilon, D$ with $0<\varepsilon<D$,

$$
I(t, x)=\left(\frac{t}{\pi}\right)^{1 / 2} \mathrm{e}^{t^{3} / 12} \sinh \left(\frac{1}{2} x t\right)\left(1+O\left(t^{-1}\right)\right), \quad t \geq 1 .
$$

Theorems 2.1 and 2.2 can be combined with (1.1) to obtain asymptotic expressions for the first passage time density. We obtain the following result for the density around zero.

Theorem 2.3. For bounded $b / \sigma^{2}>0$,

$$
f_{b}(t ; a, \sigma)=\left(\frac{b}{\sigma t \sqrt{2 \pi t}}\left(1+\frac{b t}{2 \sigma^{2}}\right) \mathrm{e}^{-b^{2} / 2 \sigma^{2} t}+O(1)\right) \mathrm{e}^{-\mathcal{F}_{1}(t) / 6 \sigma^{2}}, \quad t>0,
$$

with

$$
\mathcal{F}_{1}(t)=(t-a)^{3}+a^{3}+6 a b .
$$

The leading behavior of $f_{b}(t ; a, \sigma)$ when $t$ is small is given by

$$
\frac{b}{\sigma \sqrt{2 \pi t^{3}}} \exp \left(-\frac{a b}{\sigma^{2}}-\frac{b^{2}}{2 \sigma^{2} t}-\frac{a^{2} t}{2 \sigma^{2}}\right)
$$

We recognize (2.3) as the exact expression for the density of the time at which a Brownian motion hits a barrier $b+a t$ (see [6]). Indeed, for small $t$, the parabola $b+a t-t^{2} / 2$ is well approximated by $b+a t$. The quantity in (2.3) (ignoring the term $-a^{2} t / 2 \sigma^{2}$ ) is maximal at $t_{1}=b^{2} / 3 \sigma^{2}$ and has maximum value

$$
\left(\frac{\sigma}{b}\right)^{2}\left(\frac{27}{2 \pi}\right)^{1 / 2} \mathrm{e}^{-a b / \sigma^{2}-3 / 2}
$$

In Figure 1 we see the approximation (2.3) for the first peak, with its maximum of approximately 0.170 at $t_{1}=\frac{1}{3}$. Remember that the first peak corresponds to the scenario in which the epidemic dies out quickly. The approximations in (2.2) and (2.3) can thus be used to predict the likeliness of this scenario, and to predict the location of the first peak. For the extremely unlikely scenarios in which $t_{1}>t \rightarrow 0$, the approximation in (2.3) is sharp as well.

We next turn to approximations for the second peak.

Theorem 2.4. For bounded $b / \sigma^{2}>0$,

$$
f_{b}(t ; a, \sigma)=\frac{t^{1 / 2}}{\sigma \sqrt{2 \pi}} \sinh \left(\frac{b t}{2 \sigma^{2}}\right) \mathrm{e}^{-\mathcal{F}_{2}(t) / 6 \sigma^{2}}\left(1+O\left(t^{-1}\right)\right), \quad t \geq 1,
$$

with

$$
\mathcal{F}_{2}(t)=(t-a)^{3}-\frac{1}{4} t^{3}+a^{3}+6 a b .
$$

Noting that $\sinh x=\frac{1}{2} \mathrm{e}^{x}\left(1+O\left(\mathrm{e}^{-2 x}\right)\right), x \geq 0$, the leading behavior of $f_{b}(t ; a, \sigma)$ as $t$ becomes large is given by

$$
\frac{t^{1 / 2}}{2 \sigma \sqrt{2 \pi}} \mathrm{e}^{-\mathscr{F}_{3}(t) / 6 \sigma^{2}}
$$

with $\mathcal{F}_{3}(t)=\mathcal{F}_{2}(t)-3 b t$. The cubic $\mathcal{F}_{3}(t)$ is minimal at

$$
t_{2}=\frac{4}{3} a+\frac{2}{3} \sqrt{a^{2}+3 b}=2 a+\frac{b}{a}+O\left(\frac{b^{2}}{a^{2}}\right)
$$




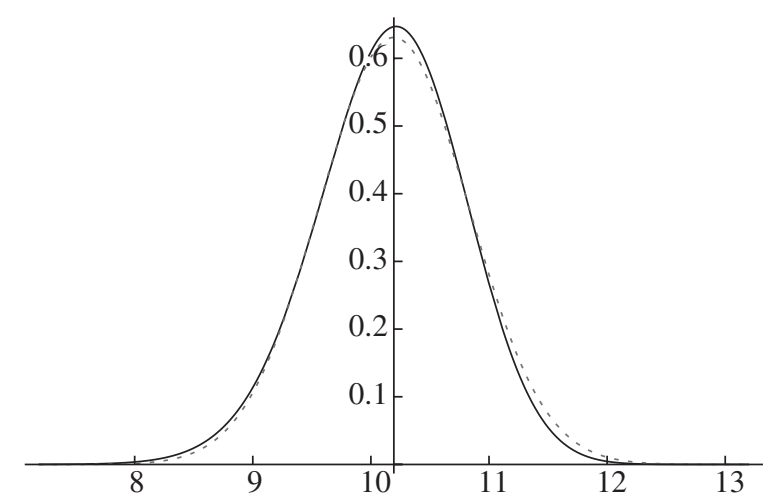

FiguRe 3: Density $f_{b}(t ; a, \sigma)$ (solid line) and approximation (2.7) (dashed line) for $a=5$ and $b=\sigma=1$.

and $\mathcal{F}_{3}\left(t_{2}\right)=-3 b^{2} / 2 a+O\left(b^{3} / a^{3}\right)$. In Figure 1, we see the approximation (2.4) for the second peak. The approximation can be used to predict the location of and the density around the second peak, but also to predict the chance of facing an extremely large epidemic. In fact, we can reformulate result (2.4) in terms of tail probabilities. A standard integration-by-parts argument yields the following result.

Corollary 2.1. (Tail final size epidemic for large $t$.) For bounded $b / \sigma^{2}>0$,

$$
\begin{aligned}
\lim _{n \rightarrow \infty} \mathrm{P}\left(K_{n} \geq t n^{2 / 3}\right) & =\int_{t}^{\infty} f_{b}\left(t^{\prime} ; a, \sigma\right) \mathrm{d} t^{\prime} \\
& =\frac{3 \sigma \sqrt{t}}{\sqrt{2 \pi}} \frac{\exp \left(-\mathcal{F}_{3}(t) / 6 \sigma^{2}\right)}{\mathcal{F}_{3}^{\prime}(t)}\left(1+O\left(t^{-1}\right)\right), \quad t \rightarrow \infty
\end{aligned}
$$

We next consider the situation when $a$ is large. We show in Section 6 that, for bounded $b$ and $a \rightarrow \infty$,

$$
t_{2}=2 a+O\left(a^{-1}\right), \quad \mathcal{F}_{3}\left(t_{2}\right)=O\left(a^{-1}\right), \quad \mathcal{F}_{3}^{\prime \prime}\left(t_{2}\right)=3 a+O\left(a^{-1}\right) .
$$

Hence, the leading behavior of $f_{b}(t ; a, \sigma)$ admits the Gaussian approximation

$$
\frac{1}{\sqrt{\pi}}\left(\frac{a}{4 \sigma^{2}}\right)^{1 / 2} \mathrm{e}^{-a\left(t-t_{2}\right)^{2} / 4 \sigma^{2}}
$$

around $t=t_{2}$. Approximation (2.7) is illustrated in Figure 3 for $a=5$ and $b=\sigma=1$.

\section{Connection with random graphs}

It is well known that the SIR model has an equivalent description in terms of the ErdôsRényi random graph [12]. The latter is a random graph on the vertex set $[n]:=\{1, \ldots, n\}$, constructed by including each of the $\left(\begin{array}{l}n \\ 2\end{array}\right)$ possible edges with probability $p$, independently of all other edges. It is well known that the components in random graphs can be determined through an exploration process (see, e.g. [2] and [19]). Take one vertex uniformly at random and label it $v(1)$. The children of $v(1)$ are all the vertices that are attached to it. Suppose that $v(1)$ has $c(1)$ children. Label these as $v(2), v(3), \ldots, v(c(1)+1)$. Now move on to $v(2)$ and explore all its children (say $c(2)$ of them) and label them as before. Note that when we explore the children 
of $v(2)$, its potential children cannot include the vertices that we have already identified. Once we finish exploring one component, we move onto the next component by choosing the starting vertex uniformly at random amongst the remaining vertices and start exploring its component. It is obvious that this constructs all the components of our graph. Write the breadth-first walk associated to this exploration process as

$$
Z_{n}(0)=0, \quad Z_{n}(i)=Z_{n}(i-1)+c(i)-1,
$$

for $i=1, \ldots, n$. For $j \geq 0$, write $\eta(j)$ as the stopping time $\eta(j)=\min \left\{i: Z_{n}(i)=-j\right\}$. The size of the $j$ th component explored in this manner is then given by $\eta(j)-\eta(j-1)$.

The following two results are due to Aldous [2].

Theorem 3.1. ([2].) Consider the breadth-first walk $Z_{n}(\cdot)$ exploring the components of the random graph $G(n, p)$. For $p=\left(1+a n^{-1 / 3}\right) / n, a \in \mathbb{R}$ fixed, and $n \rightarrow \infty$,

$$
n^{-1 / 3} Z_{n}\left(\left\lfloor n^{2 / 3} t\right\rfloor\right) \stackrel{\mathrm{D}}{\rightarrow} W_{t}^{a},
$$

in the sense of convergence in the $J_{1}$ Skorokhod topology on the space of right-continuous left-limited functions on $\mathbb{R}^{+}$, where $\left(W_{t}^{a}\right)_{t \geq 0}$ is the process $\left(W_{t}+a t-t^{2} / 2\right)_{t \geq 0}$.

Let $\mathcal{C}_{n}^{i}(a)$ denote the component in $G(n, p)$ of the $i$ th largest order (where ties are broken arbitrarily).

Theorem 3.2. ([2].) For $p=\left(1+a n^{-1 / 3}\right) / n, a \in \mathbb{R}$ fixed, and $n \rightarrow \infty$,

$$
\left(\left|\mathcal{C}_{n}^{1}(a)\right| n^{-2 / 3},\left|\mathcal{C}_{n}^{2}(a)\right| n^{-2 / 3}, \ldots\right) \stackrel{\mathrm{D}}{\rightarrow}\left(C_{1}(a), C_{2}(a), \ldots\right),
$$

where $C_{1}(a)>C_{2}(a)>\cdots$ are the ordered excursions of the reflected version of the process $\left(W_{t}^{a}\right)_{t \geq 0}$.

Theorem 3.2 says that the ordered connected components in the critical Erdős-Rényi random graph are described by the ordered excursions of the reflected version of $\left(W_{t}^{a}\right)_{t \geq 0}$, the same limit process that occurs in the SIR model. Note that this process starts at 0 , and, hence, does not depend on $b$. For $a>0$, the process might have several small excursions before it takes off for a long excursion and the giant component in $G(n, p)$ starts to form. The component keeps growing until the time at which the process intersects the $t$-axis, and after that, the strong negative drift of the parabola makes it unlikely for more large components to occur.

This critical behavior of Erdős-Rényi random graphs has received tremendous attention over the past decades. We refer the reader to [2], [4], [11], and the references therein. In [17] an exact formula was derived for the distribution function of the limiting variable $C_{1}(a)$ (of the largest component). In [2] it was shown that the limit in (3.1) is in fact a certain multiplicative coalescent, and in [10] the limit process was treated as a point process. In [21] a local limit theorem was derived for the joint tail behavior of several of the largest connected components.

Let us now further discuss how results for the largest component $C_{1}(a)$ can be obtained from results for $f_{b}(t ; a, \sigma)$. Let $h_{a}(t)$ denote the density of $C_{1}(a)$, and let

$$
H_{a}(t)=\int_{0}^{t} h_{a}(s) \mathrm{d} s=\mathrm{P}\left(C_{1}(a) \leq t\right), \quad t \geq 0,
$$

denote the distribution function. It is clear that $H_{a}(t)$ decreases in $a \in \mathbb{R}$. 
In the SIR model the critical threshold for the initial number of vertices to be explored turned out to be $n^{1 / 3}$, and, in fact, the final size of the epidemic follows from exploring the connections of each of the $b n^{1 / 3}$ vertices. Let us first present the connection between exploring $b n^{1 / 3}$ vertices and exploring an arbitrary vertex (the first component) in the random graph.

Let $\mathcal{C}(v)$ be the component that contains the vertex $v$. Throughout the proof of Lemmas 3.1 and 3.2, we will make use of the fact that there is a constant $c_{a}$ such that, for all $n \geq k \geq 1$,

$$
\mathrm{P}(|\mathcal{C}(1)| \geq k) \leq \frac{c_{a}}{k^{1 / 2}}, \quad \mathrm{E}[|\mathcal{C}(1)|] \leq c_{a} n^{1 / 3}, \quad \mathrm{E}\left[|\mathcal{C}(1)| \mathbf{1}_{\{|\mathcal{C}(1)| \leq k\}}\right] \leq c_{a} k^{1 / 2}
$$

These bounds can be retrieved from various sources (see, e.g. [4] and [11]); they also follow from [16, Theorem 1] (see also the references in [16] for a detailed account of the history of such results).

In the following, we let $b_{n}=b n^{1 / 3}$.

Lemma 3.1. (Clusters are, with high probability, disjoint.) We have

$$
\mathrm{P}\left(\left|\bigcup_{i=1}^{b_{n}} \mathcal{C}(i)\right|>t n^{2 / 3}\right)=\mathrm{P}\left(\sum_{i=1}^{b_{n}}|\mathcal{C}(i)|>t n^{2 / 3}\right)+O\left(b^{2}\right),
$$

uniformly in $n$.

Proof. The upper bound in (3.4) is immediate, since $\sum_{i=1}^{b_{n}}|\mathcal{C}(i)| \geq\left|\bigcup_{i=1}^{b_{n}} \mathcal{C}(i)\right|$. For the lower bound, we note that, conditionally on the event that the sets of vertices $\mathcal{C}(i)$ are all disjoint, we have

$$
\left\{\left|\bigcup_{i=1}^{b_{n}} \mathcal{C}(i)\right|>t n^{2 / 3}\right\}=\left\{\sum_{i=1}^{b_{n}}|\mathcal{C}(i)|>t n^{2 / 3}\right\}
$$

As a result,

$$
\begin{aligned}
\mathrm{P}\left(\sum_{i=1}^{b_{n}}|\mathcal{C}(i)|>t n^{2 / 3}\right)-\mathrm{P}\left(\left|\bigcup_{i=1}^{b_{n}} \mathcal{C}(i)\right|>t n^{2 / 3}\right) & \leq \mathrm{P}\left(\text { there exists } i \neq j \in\left[b_{n}\right]: i \rightarrow j\right) \\
& \leq b_{n}^{2} \mathrm{P}(i \rightarrow j) \\
& =b_{n}^{2} \frac{\mathrm{E}[|\mathcal{C}(i)|]}{n}
\end{aligned}
$$

By the second bound in (3.3),

$$
\mathrm{P}\left(\sum_{i=1}^{b_{n}}|\mathcal{C}(i)|>t n^{2 / 3}\right)-\mathrm{P}\left(\left|\bigcup_{i=1}^{b_{n}} \mathcal{C}(i)\right|>t n^{2 / 3}\right) \leq c_{a} b^{2}=O\left(b^{2}\right) .
$$

Lemma 3.2. (Clusters have a heavy-tailed distribution.) Uniformly in $n$ and $b>0$, for any $\varepsilon>0$,

$$
\begin{aligned}
b_{n} \mathrm{P}\left(|\mathcal{C}(1)|>t n^{2 / 3}\right)+O\left(b^{2}\right) & \leq \mathrm{P}\left(\sum_{i=1}^{b_{n}}|\mathcal{C}(i)|>t n^{2 / 3}\right) \\
& \leq b_{n} \mathrm{P}\left(|\mathcal{C}(1)|>(t-\varepsilon) n^{2 / 3}\right)+O\left(\frac{b^{2}}{\varepsilon}\right)+O(b \sqrt{\varepsilon}) .
\end{aligned}
$$


Proof. We start with the lower bound. For this, we note that, by inclusion-exclusion,

$$
\begin{aligned}
& \sum_{i=1}^{b_{n}} \mathrm{P}\left(|\mathcal{C}(i)|>t n^{2 / 3}\right)-\sum_{1 \leq i<j \leq b_{n}} \mathrm{P}\left(|\mathcal{C}(i)|>t n^{2 / 3},|\mathcal{C}(j)|>t n^{2 / 3}\right) \\
& \quad \leq \mathrm{P}\left(\bigcup_{i=1}^{b_{n}}\left\{|\mathcal{C}(i)|>t n^{2 / 3}\right\}\right) \\
& \quad \leq \mathrm{P}\left(\sum_{i=1}^{b_{n}}|\mathcal{C}(i)|>t n^{2 / 3}\right)
\end{aligned}
$$

The first probability equals $b_{n} \mathrm{P}\left(|\mathcal{C}(1)|>t n^{2 / 3}\right)$. The second probability can be split according to whether or not $j \in \mathcal{C}(i)$. When $j \in \mathcal{C}(i)$, by the second bound in (3.3) we obtain an upper bound,

$$
\frac{b_{n}^{2}}{n} \mathrm{E}[|\mathcal{C}(1)|]=O\left(b^{2}\right)
$$

When $j \notin \mathcal{C}(i)$, we obtain

$$
\mathrm{P}\left(|\mathcal{C}(i)|>t n^{2 / 3},|\mathcal{C}(j)|>t n^{2 / 3}, j \notin \mathcal{C}(i)\right) \leq \mathrm{P}\left(|\mathcal{C}(i)|>t n^{2 / 3}\right) \mathrm{P}\left(|\mathcal{C}(j)|>t n^{2 / 3}\right),
$$

so that this contribution can be bounded, using the first bound in (3.3), by, for each $t>0$ fixed,

$$
b_{n}^{2} \mathrm{P}\left(|\mathcal{C}(i)|>t n^{2 / 3}\right)^{2}=O\left(b^{2}\right) .
$$

This proves the lower bound. For the upper bound, we must be more careful. We first split the probability according to how many $i \in\left[b_{n}\right]$ there are for which $\left\{|\mathcal{C}(i)|>\varepsilon n^{2 / 3}\right\}$. Denote this number by $I$. We bound the contribution from $I=0$ using the Markov inequality and the third bound in (3.3):

$$
\begin{aligned}
\mathrm{P}\left(\sum_{i=1}^{b_{n}}|\mathcal{C}(i)|>t n^{2 / 3}, I=0\right) & \leq \mathrm{P}\left(\sum_{i=1}^{b_{n}}|\mathcal{C}(i)| \mathbf{1}_{\left\{|\mathcal{C}(i)| \leq \varepsilon n^{2 / 3}\right\}}>t n^{2 / 3}\right) \\
& \leq \frac{b_{n}}{t n^{2 / 3}} \mathrm{E}\left[|\mathcal{C}(i)| \mathbf{1}_{\left\{|\mathcal{C}(i)| \leq \varepsilon n^{2 / 3}\right\}}\right] \\
& \leq \frac{C b_{n}}{t n^{2 / 3}} \sqrt{\varepsilon} n^{1 / 3} \\
& =O(\sqrt{\varepsilon} b) .
\end{aligned}
$$

The contribution from $I \geq 2$ can be bounded from above by the first bound in (3.3):

$$
b_{n}^{2} \mathrm{P}\left(|\mathcal{C}(1)|>\varepsilon n^{2 / 3},|\mathcal{C}(2)|>\varepsilon n^{2 / 3}\right) \leq O\left(\frac{b_{n}^{2}}{\varepsilon n^{2 / 3}}\right)=O\left(\frac{b^{2}}{\varepsilon}\right) .
$$

Therefore, the main contribution comes from $I=1$, which equals

$$
b_{n} \mathrm{P}\left(\sum_{i=1}^{b_{n}}|\mathcal{C}(i)|>t n^{2 / 3},|\mathcal{C}(1)|>\varepsilon n^{2 / 3},|\mathcal{C}(i)| \leq \varepsilon n^{2 / 3} \text { for all } i \in\left\{2, \ldots, b_{n}\right\}\right) .
$$


We finally split this probability according to whether or not $|\mathcal{C}(1)| \geq(t-\varepsilon) n^{2 / 3}$. The contribution due to $|\mathcal{C}(1)| \geq(t-\varepsilon) n^{2 / 3}$ is bounded by $b_{n} \mathrm{P}\left(|\mathcal{C}(1)|>(t-\varepsilon) n^{2 / 3}\right)$. When $|\mathcal{C}(1)|<(t-\varepsilon) n^{2 / 3}$, then we must have $\sum_{i=2}^{b_{n}}|\mathcal{C}(i)|>\varepsilon n^{2 / 3}$, so that, again by the Markov inequality, this contribution is bounded by

$$
\begin{aligned}
\frac{b_{n}}{\varepsilon n^{2 / 3}} & \mathrm{E}\left[\sum_{i=2}^{b_{n}}|\mathcal{C}(i)| \mathbf{1}_{\left\{|\mathcal{C}(i)| \leq \varepsilon n^{2 / 3},|\mathcal{C}(1)|>\varepsilon n^{2 / 3}\right\}}\right] \\
& =\frac{b^{2}}{\varepsilon} \mathrm{E}\left[|\mathcal{C}(2)| \mathbf{1}_{\left\{|\mathcal{C}(2)| \leq \varepsilon n^{2 / 3},|\mathcal{C}(1)|>\varepsilon n^{2 / 3}\right\}}\right] \\
& \leq \frac{b^{2}}{\varepsilon} \mathrm{E}\left[|\mathcal{C}(2)| \mathbf{1}_{\left\{|\mathcal{C}(2)| \leq \varepsilon n^{2 / 3}\right\}}\right] \mathrm{P}\left(|\mathcal{C}(1)|>\varepsilon n^{2 / 3}\right) \\
& =O\left(\frac{b^{2}}{\varepsilon}\right),
\end{aligned}
$$

using the first and third bounds in (3.3). Summing all contributions proves the upper bound.

Theorem 3.3. (The cluster tail.) With $b_{n}=\left\lceil b n^{1 / 3}\right\rceil$,

$$
\begin{aligned}
\lim _{n \rightarrow \infty} n^{1 / 3} \mathrm{P}\left(|\mathcal{C}(1)|>t n^{2 / 3}\right) & =\lim _{b \downarrow 0} \frac{1}{b} \lim _{n \rightarrow \infty} \mathrm{P}\left(\left|\bigcup_{i=1}^{b_{n}} \mathcal{C}(i)\right|>t n^{2 / 3}\right) \\
& =\lim _{b \downarrow 0} \frac{1}{b} \int_{t}^{\infty} f_{b}(s ; a, \sigma) \mathrm{d} s .
\end{aligned}
$$

Proof. Combining Lemmas 3.1 and 3.2, we obtain, for any $\varepsilon>0$,

$$
\begin{aligned}
b_{n} \mathrm{P}\left(|\mathcal{C}(1)|>t n^{2 / 3}\right)+O\left(b^{2}\right) & \leq \mathrm{P}\left(\left|\bigcup_{i=1}^{b_{n}} \mathcal{C}(i)\right|>t n^{2 / 3}\right) \\
& \leq b_{n} \mathrm{P}\left(|\mathcal{C}(1)|>(t-\varepsilon) n^{2 / 3}\right)+O\left(\frac{b^{2}}{\varepsilon}\right)+O(b \sqrt{\varepsilon}) .
\end{aligned}
$$

By Theorem 1.1 and the relation between the final size of an SIR epidemic and the Erdôs-Rényi random graph discussed above,

$$
\lim _{n \rightarrow \infty} \mathrm{P}\left(\left|\bigcup_{i=1}^{b_{n}} \mathcal{C}(i)\right|>t n^{2 / 3}\right)=\int_{t}^{\infty} f_{b}(s ; a, \sigma) \mathrm{d} s .
$$

As a result, we obtain

$$
\begin{aligned}
& \lim _{n \rightarrow \infty} b_{n} \mathrm{P}\left(|\mathcal{C}(1)|>t n^{2 / 3}\right)+O\left(b^{2}\right) \\
& \quad \leq \int_{t}^{\infty} f_{b}(s ; a, \sigma) \mathrm{d} s \\
& \quad \leq \lim _{n \rightarrow \infty} b_{n} \mathrm{P}\left(|\mathcal{C}(1)|>(t-\varepsilon) n^{2 / 3}\right)+O\left(\frac{b^{2}}{\varepsilon}\right)+O(b \sqrt{\varepsilon}) .
\end{aligned}
$$


Divide by $b$ and let $b \downarrow 0$ to obtain

$$
\begin{aligned}
\lim _{n \rightarrow \infty} n^{1 / 3} \mathrm{P}\left(|\mathcal{C}(1)|>t n^{2 / 3}\right) & \leq \lim _{b \downarrow 0} \int_{t}^{\infty} \frac{1}{b} f_{b}(s ; a, \sigma) \mathrm{d} s \\
& \leq \lim _{n \rightarrow \infty} n^{1 / 3} \mathrm{P}\left(|\mathcal{C}(1)|>(t-\varepsilon) n^{2 / 3}\right)+O(\sqrt{\varepsilon}) .
\end{aligned}
$$

By dominated convergence,

$$
\lim _{b \downarrow 0} \int_{t}^{\infty} \frac{1}{b} f_{b}(s ; a, \sigma) \mathrm{d} s=\int_{t}^{\infty} g_{a}(t ; \sigma) \mathrm{d} s,
$$

where $g_{a}(t ; \sigma)=\lim _{b \downarrow 0} f_{b}(t ; a, \sigma) / b$. From (1.1), together with the fact that $\operatorname{Ai}(u) \operatorname{Bi}^{\prime}(u)-$ $\operatorname{Ai}^{\prime}(u) \operatorname{Bi}(u)$ is the Wronskian of the Airy equation, and is thus identically equal to $1 / \pi[1$, Section 10.4.10], we see that

$$
g_{a}(t ; \sigma)=\lim _{b \downarrow 0} \frac{1}{b} f_{b}(t ; a, \sigma)=\frac{1}{\sigma^{2}} \mathrm{e}^{-\left((t-a)^{3}+a^{3}\right) / 6 \sigma^{2}} \int_{-\infty}^{\infty} \frac{\mathrm{e}^{t u / c}}{\pi^{2}\left(\mathrm{Ai}^{2}(u)+\mathrm{Bi}^{2}(u)\right)} \mathrm{d} u,
$$

which is integrable on $[t, \infty)$ for all $t>0$. Therefore, (3.6) implies that

$$
\lim _{n \rightarrow \infty} n^{1 / 3} \mathrm{P}\left(|\mathcal{C}(1)|>t n^{2 / 3}\right)=\int_{t}^{\infty} g_{a}(s ; \sigma) \mathrm{d} s .
$$

This proves the result.

By [21, Lemma 2.2] we have, for some function $s \mapsto \Phi(s ; a)$,

$$
\lim _{n \rightarrow \infty} n \mathrm{P}\left(|\mathcal{C}(1)|=\left\lceil t n^{2 / 3}\right\rceil\right)=t \Phi(t ; a),
$$

which identifies $s \Phi(s ; a)=g_{a}(s ; \sigma)=\lim _{b \downarrow 0} f_{b}(s ; a, \sigma) / b$. The function $g_{a}(t)=g_{a}(t ; 1)$ plays a rather special role in the study of the largest component. It holds (see, e.g. [13] and [21] and recall (3.2)) that

$$
h_{a}(t)=\frac{1}{t} g_{a}(t) H_{a-t}(t), \quad t \geq 0, a \in \mathbb{R} .
$$

Informally, (3.8) can be understood as follows. In order for the largest cluster to have size $t n^{2 / 3}$, we can multiply $n / t n^{2 / 3}$ times the probability that the cluster of vertex 1 has size $t n^{2 / 3}$, and the remaining clusters in the graph of size $n-t n^{2 / 3}$ have size at most $t n^{2 / 3}$. Since

$$
p=\frac{1}{n}\left(1+a n^{-1 / 3}\right)=\frac{1}{n-t n^{2 / 3}}\left(1+(a-t)\left(n-t n^{2 / 3}\right)^{-1 / 3}\right)+o\left(n^{-4 / 3}\right),
$$

the latter has probability approximately $H_{a-t}(t)$.

A more common form in which $g_{a}(t)$ is given is

$$
g_{a}(t)=\mathrm{e}^{-\left((t-a)^{3}+a^{3}\right) / 6} \frac{G\left(t^{3 / 2}\right)}{\sqrt{2 \pi}},
$$

where $G(s)=\sum_{k=-1}^{\infty} \rho_{k} s^{k}$ and the $\rho_{k}$ are the Wright constants that arise in graph enumeration (see [13], [17], and [21]). The overview paper [9] contains many results on the Wright constants, and their relation to the Brownian excursion area and Airy functions. Denote by 
$a_{j}=-\left|a_{j}\right|, j=1,2, \ldots$, the zeros of the Airy function $\operatorname{Ai}(z)$ such that $0<\left|a_{1}\right|<\left|a_{2}\right|<\cdots$; hence,

$$
\operatorname{Ai}\left(a_{j}\right)=\operatorname{Ai}\left(-\left|a_{j}\right|\right)=0, \quad j=1,2, \ldots
$$

We have (see [9, Equation (80)])

$$
G(s)=-\sqrt{2 \pi} \sum_{j=1}^{\infty} \exp \left(-2^{-1 / 3}\left|a_{j}\right|(-s)^{2 / 3}\right), \quad s<0 .
$$

From (3.7) and (3.9), we instead obtain

$$
G\left(t^{3 / 2}\right)=\frac{\sqrt{2 \pi}}{\pi^{2}} \int_{-\infty}^{\infty} \frac{\exp \left(t u 2^{-1 / 3}\right)}{\mathrm{Ai}^{2}(u)+\mathrm{Bi}^{2}(u)} \mathrm{d} u, \quad t>0,
$$

which seems to be a new expression for the function $G$. To make the connection to the excursion area, we note that, by [9, Equation (36)] and using the notation in [9, Equation (79)],

$$
G(s)=\frac{1}{s} \mathrm{E}\left[\mathrm{e}^{s \mathcal{B}_{\mathrm{ex}}}\right] \equiv \frac{1}{s} \psi_{\mathrm{ex}}(-s),
$$

where $\mathcal{B}_{\mathrm{ex}}$ is the normalized excursion area of Brownian motion given by

$$
\mathcal{B}_{\mathrm{ex}}=\int_{0}^{1} B_{\mathrm{ex}}(t) \mathrm{d} t
$$

and $\left(B_{\mathrm{ex}}(t)\right)_{t \in[0,1]}$ is the normalized Brownian excursion [9, Equation (1-2)]. Then, we arrive at the following corollary to Theorem 3.3.

Corollary 3.1. (Excursion area moment generating function.) There is the representation

$$
\mathrm{E}\left[\mathrm{e}^{t \mathcal{B}_{\mathrm{ex}}}\right]=\psi_{\mathrm{ex}}(-t)=t \frac{\sqrt{2 \pi}}{\pi^{2}} \int_{-\infty}^{\infty} \frac{\exp \left(t^{2 / 3} u 2^{-1 / 3}\right)}{\mathrm{Ai}^{2}(u)+\mathrm{Bi}^{2}(u)} \mathrm{d} u,
$$

which is absolutely convergent when $|\arg (t)|<3 \pi / 4$, when we take the principal value of $t^{2 / 3}$.

While [9] gives a wealth of results concerning $\psi_{\mathrm{ex}}(-t)$, we have not been able to find this explicit formula for $\psi_{\mathrm{ex}}(-t)$ in the literature.

The asymptotic results in Section 2 on the left and right tails of $f_{b}(t ; a, \sigma)$ can also be obtained for $g_{a}(t)$. More specifically, we have

$$
\frac{1}{\pi^{2}} \int_{-\infty}^{\infty} \frac{\mathrm{e}^{t u / 2^{1 / 3}}}{\mathrm{Ai}^{2}(u)+\mathrm{Bi}^{2}(u)} \mathrm{d} u=\frac{t^{-3 / 2}}{\sqrt{2 \pi}}+O(1), \quad t \downarrow 0,
$$

and

$$
\frac{1}{\pi^{2}} \int_{-\infty}^{\infty} \frac{\mathrm{e}^{t u / 2^{1 / 3}}}{\mathrm{Ai}^{2}(u)+\mathrm{Bi}^{2}(u)} \mathrm{d} u=\frac{t^{3 / 2} \mathrm{e}^{t^{3} / 24}}{(8 \pi)^{1 / 2}}\left(1+O\left(t^{-3 / 2}\right)\right), \quad t \rightarrow \infty
$$

From (3.7) and (3.12), we obtain

$$
\frac{1}{t} g_{a}(t)=\frac{t^{1 / 2}}{(8 \pi)^{1 / 2}} \exp \left(-\frac{1}{8} t(t-2 a)^{2}\right)\left(1+O\left(t^{-3 / 2}\right)\right), \quad t \rightarrow \infty .
$$


Lemma 3.3. (Tail bounds on the largest connected component distribution.) We have

$$
1-\int_{t}^{\infty} \frac{1}{s} g_{a}(s) \mathrm{d} s \leq H_{a}(t) \leq \exp \left(-\int_{t}^{\infty} \frac{1}{s} g_{a}(s) \mathrm{d} s\right), \quad t>0 .
$$

Proof. We have, from (3.8),

$$
1-H_{a}(t)=\int_{t}^{\infty} \frac{1}{s} g_{a}(s) H_{a-s}(s) \mathrm{d} s \leq \int_{t}^{\infty} \frac{1}{s} g_{a}(s) \mathrm{d} s,
$$

which is the first inequality in (3.14). Next, we have, from (3.8),

$$
\frac{H_{a}^{\prime}(t)}{H_{a}(t)}=\frac{1}{t} g_{a}(t) \frac{H_{a-t}(t)}{H_{a}(t)}, \quad t>0,
$$

and so, by integration from $t$ to $\infty$, using $H_{a}(\infty)=1$,

$$
H_{a}(t)=\exp \left(-\int_{t}^{\infty} \frac{1}{s} g_{a}(s) \frac{H_{a-s}(s)}{H_{a}(s)} \mathrm{d} s\right), \quad t>0 .
$$

We have $H_{a-s}(s) / H_{a}(s) \geq 1, s>0$, since $H_{a}(t)$ decreases in $a$. This gives the second inequality in (3.14).

Theorem 3.4. (Maximal cluster tail for large $t$.) We have

$$
1-H_{a}(t)=8 \frac{t^{1 / 2} \exp \left(-t(t-2 a)^{2} / 8\right)}{\sqrt{8 \pi}(t-2 a)(3 t-2 a)}\left(1+O\left(t^{-3 / 2}\right)\right), \quad t \rightarrow \infty .
$$

Proof. From (3.14) we see that

$$
1-H_{a}(t)=\int_{t}^{\infty} \frac{1}{s} g_{a}(s) \mathrm{d} s+O\left(\left(\int_{t}^{\infty} \frac{1}{s} g_{a}(s) \mathrm{d} s\right)^{2}\right) .
$$

Now, by integrating by parts, noting that $\mathrm{d}\left[s(s-2 a)^{2}\right] / \mathrm{d} s=(s-2 a)(3 s-2 a)$, we obtain, from (3.13),

$$
\begin{aligned}
\int_{t}^{\infty} \frac{1}{s} g_{a}(s) \mathrm{d} s & =\int_{t}^{\infty} \frac{s^{1 / 2} \exp \left(-s(s-2 a)^{2} / 8\right)}{(8 \pi)^{1 / 2}}\left(1+O\left(t^{-3 / 2}\right)\right) \mathrm{d} s \\
& =8 \frac{t^{1 / 2} \exp \left(-t(t-2 a)^{2} / 8\right)}{\sqrt{8 \pi}(t-2 a)(3 t-2 a)}\left(1+O\left(t^{-3 / 2}\right)\right), \quad t \rightarrow \infty
\end{aligned}
$$

which yields (3.15).

Theorem 3.4 sharpens [17, Equation (1.12)].

To obtain the asymptotics of $H_{a}(t)$ as $t \downarrow 0$ by relatively elementary means is harder. This is due to the fact that replacing $H_{a-s}(s) / H_{a}(s)$ by 1 , as was done in the proof of the second inequality in (3.14), is a rather crude operation when $s$ is close to 0 . Alternatively, we have, from [17, Equation (4.28)],

$$
\frac{H_{a-s}(s)}{H_{a}(s)}=\mathrm{e}^{\mu}\left(1+O\left(s^{1 / 2}\right)\right), \quad s \downarrow 0,
$$


where $\mu=0.854033 \ldots$ is the root of $\mu^{-1 / 2} \mathrm{e}^{\mu}=\int_{0}^{\mu} t^{-1 / 2} \mathrm{e}^{t} \mathrm{~d} t$. Thus, combining (3.11), (3.14), and (3.16), we obtain

$$
H_{a}(t)=\exp \left(-\frac{2}{3} \frac{t^{-3 / 2} \mathrm{e}^{\mu}}{\sqrt{2 \pi}}+O\left(t^{-1}\right)\right), \quad t \downarrow 0 .
$$

Hence, identity (3.8), together with the asymptotic evaluation of the function $g_{a}(t)$, suffices to derive large- $t$ asymptotics for $H_{a}(t)$, but does not give enough information to obtain small- $t$ asymptotics. Indeed, in order to estimate the left tail of $H_{a}(t)$, and to derive for instance (3.16) and (3.17), a different approach is needed. Pittel [17] obtained these results by deriving an explicit integral formula for $H_{a}(t)$ (see [17, Equation (1.7)]).

\section{Discussion}

\subsection{Scaling properties}

The results in this paper for the hitting time of a Brownian motion with parabolic drift obey simple scaling properties. Define the process $\left(V_{\sigma, \kappa}^{a}(t)\right)_{t \geq 0}$ as

$$
V_{\sigma, \kappa}^{a}(t)=\sigma W_{t}+a t-\kappa \frac{1}{2} t^{2}
$$

Fix $\tau>0$, and note that $\left(W_{\tau^{2} t}\right)_{t \geq 0}$ has the same distribution as $\left(\tau W_{t}\right)_{t \geq 0}$. We obtain the relation

$$
V_{\sigma, \kappa}^{a}(t) \stackrel{\mathrm{D}}{=} \frac{\sigma}{\tau}\left(W_{\tau^{2} t}+\frac{a}{\sigma \tau} \tau^{2} t-\frac{\kappa}{\sigma \tau^{3}} \frac{\left(\tau^{2} t\right)^{2}}{2}\right) \stackrel{\mathrm{D}}{=} \frac{\sigma}{\tau} V_{1, \kappa /\left(\sigma \tau^{3}\right)}^{a /(\sigma \tau)}\left(\tau^{2} t\right) .
$$

Choosing $\tau=(\kappa / \sigma)^{1 / 3}$ gives

$$
V_{\sigma, \kappa}^{a}(t) \stackrel{\mathrm{D}}{=} \frac{\sigma^{4 / 3}}{\kappa^{1 / 3}} V_{1,1}^{a \kappa^{-1 / 3} \sigma^{-2 / 3}}\left(\kappa^{2 / 3} \sigma^{-2 / 3} t\right)
$$

Let $T_{\sigma, \kappa}^{a}(b)$ denote the first time the process $\left(V_{\sigma, \kappa}^{a}(t)\right)_{t \geq 0}$ hits level $-b$. Then

$$
T_{\sigma, \kappa}^{a}(b)=\kappa^{-2 / 3} \sigma^{2 / 3} T_{1,1}^{a \kappa^{-1 / 3} \sigma^{-2 / 3}}\left(b \kappa^{1 / 3} \sigma^{-4 / 3}\right) .
$$

The hitting time $T_{\sigma, \kappa}^{a}(b)$, for specific choices of $\sigma, a$, and $\kappa$, occurs in the study of SIR models with general sampling procedures [14], SIR models with vaccination [7] and inhomogeneous random graphs [3], [20]. Our results for the simple SIR model and the Erdôs-Rényi (homogeneous) random graph thus extend to these more general settings via the scaling relation (4.1).

\subsection{The Brownian excursion area}

Formula (3.10) gives a new expression for the moment generating function of the Brownian excursion area $\mathcal{B}_{\text {ex }}$. Since (3.10) holds on the imaginary axis (except at 0 ), this also gives the Fourier transform of $\mathscr{B}_{\mathrm{ex}}$. We leave retrieving the density of $\mathscr{B}_{\mathrm{ex}}$ by Fourier inversion as an open problem.

\subsection{Asymptotics for multiple large clusters}

Another topic for future research is to determine asymptotic expressions like in Theorem 3.4, but then for the joint tail behavior of several of the largest connected components. The local limit theorem for the largest $k$ connected components as derived in [21], for each $k \geq 1$, could be the starting point for this analysis. This local limit is given in terms of the same functions appearing in the local limit theorem for the largest connected component, as given in (3.8). 


\section{Proof of Theorem 2.1}

We rely heavily on the approximations (see [1, Sections 10.4.59-60 and 10.4.63-64])

$$
\begin{aligned}
& \operatorname{Ai}(u)=\frac{u^{-1 / 4}}{2 \sqrt{\pi}} \mathrm{e}^{-2 u^{3 / 2} / 3}\left(1-\frac{5}{48} u^{-3 / 2}+O\left(u^{-3}\right)\right), \quad u>0, \\
& \operatorname{Bi}(u)=\frac{u^{-1 / 4}}{\sqrt{\pi}} \mathrm{e}^{2 u^{3 / 2} / 3}\left(1+\frac{5}{48} u^{-3 / 2}+O\left(u^{-3}\right)\right), \quad u>0,
\end{aligned}
$$

and, again, for $u>0$,

$$
\begin{aligned}
& \operatorname{Ai}(-u)=\frac{u^{-1 / 4}}{\sqrt{\pi}}\left(\sin \left(\frac{2}{3} u^{3 / 2}+\frac{\pi}{4}\right)-\frac{5}{48} u^{-3 / 2} \cos \left(\frac{2}{3} u^{3 / 2}+\frac{\pi}{4}\right)+O\left(u^{-3}\right)\right), \\
& \operatorname{Bi}(-u)=\frac{u^{-1 / 4}}{\sqrt{\pi}}\left(\cos \left(\frac{2}{3} u^{3 / 2}+\frac{\pi}{4}\right)+\frac{5}{48} u^{-3 / 2} \sin \left(\frac{2}{3} u^{3 / 2}+\frac{\pi}{4}\right)+O\left(u^{-3}\right)\right) .
\end{aligned}
$$

Let us first study the contribution of the positive integration range $u \geq 0$ in (2.1). The integrand is estimated in (6.1) below for $u>2 x$ and is easily seen to be $O\left(\mathrm{e}^{t u} / \mathrm{Bi}(u)\right)$ when $0 \leq u \leq 2 x$ uniformly in $x>0$. Therefore, the contribution of the range $u>0$ is uniformly bounded in $x \in[0, D], t \in[0, T]$, for any $D>0$ and $T>0$.

Now we turn to the contribution of the negative integration range $u \leq 0$ in (2.1). From (5.3) and (5.4), we have

$$
\begin{aligned}
& \operatorname{Ai}(-u-x) \operatorname{Bi}(-u)-\operatorname{Ai}(-u) \operatorname{Bi}(-u-x) \\
& \quad=\frac{1}{\pi u^{1 / 4}(u+x)^{1 / 4}}\left(\sin \left(\frac{2}{3}(u+x)^{3 / 2}-\frac{2}{3} u^{3 / 2}\right)+O\left(u^{-3 / 2}\right)\right) .
\end{aligned}
$$

Also,

$$
\mathrm{Ai}^{2}(-u)+\mathrm{Bi}^{2}(-u)=\frac{1}{\pi \sqrt{u}}\left(1+O\left(u^{-3 / 2}\right)\right) .
$$

This leads to the approximation

$$
\begin{aligned}
& \frac{\operatorname{Bi}(-u) \operatorname{Ai}(-u-x)-\operatorname{Ai}(-u) \operatorname{Bi}(-u-x)}{\pi\left(\mathrm{Ai}^{2}(-u)+\mathrm{Bi}^{2}(-u)\right)} \\
& =\frac{1}{\pi}\left(\frac{u}{u+x}\right)^{1 / 4}\left(\sin \left(\frac{2}{3}(u+x)^{3 / 2}-\frac{2}{3} u^{3 / 2}\right)+O\left(u^{-3 / 2}\right)\right) .
\end{aligned}
$$

Now we use

$$
\begin{aligned}
\frac{2}{3}(u+x)^{3 / 2}-\frac{2}{3} u^{3 / 2} & =x \sqrt{u}+\frac{x^{2}}{4 \sqrt{u}}-\frac{x^{3}}{24 u^{3 / 2}}+\cdots, \\
\left(\frac{u}{u+x}\right)^{1 / 4} & =1-\frac{x}{4 u}+\frac{5}{32} \frac{x^{2}}{u^{2}}-\cdots
\end{aligned}
$$

to obtain

$$
\begin{aligned}
& \frac{\operatorname{Bi}(-u) \operatorname{Ai}(-u-x)-\operatorname{Ai}(-u) \operatorname{Bi}(-u-x)}{\pi\left(\mathrm{Ai}^{2}(-u)+\mathrm{Bi}^{2}(-u)\right)} \\
& =\frac{1}{\pi}\left(1-\frac{x+x^{4} / 8}{4 u}\right) \sin (x \sqrt{u})+\frac{x^{2}}{4 \pi \sqrt{u}} \cos (x \sqrt{u})+R(u, x) .
\end{aligned}
$$


Here the remainder $R(u, x)=O\left(u^{-3 / 2}\right)$ as $u \rightarrow \infty$ and $O\left(u^{-1 / 2}\right)$ as $u \rightarrow 0$, since the first member of (5.6) is bounded at $u=0$, uniformly in $x \in(0, D]$. As a consequence, the contribution of $R(u, x)$ to the integral is $O(1)$ for $x \in(0, D]$.

Using partial integration, we find that

$$
\frac{1}{\pi} \int_{0}^{\infty} \mathrm{e}^{-t u} \sin (x \sqrt{u}) \mathrm{d} u=\frac{x}{2 \pi t}\left(\frac{\pi}{t}\right)^{1 / 2} \mathrm{e}^{-x^{2} / 4 t}
$$

and

$$
\frac{x^{2}}{4 \pi} \int_{0}^{\infty} \mathrm{e}^{-t u} \frac{\cos (x \sqrt{u})}{\sqrt{u}} \mathrm{~d} u=\frac{x^{2}}{4 \pi}\left(\frac{\pi}{t}\right)^{1 / 2} \mathrm{e}^{-x^{2} / 4 t} .
$$

Furthermore,

$$
\int_{0}^{\infty} \frac{\sin (x \sqrt{u})}{u} \mathrm{e}^{-t u} \mathrm{~d} u=2 \int_{0}^{\infty} \frac{\sin y}{y} \mathrm{e}^{-\varepsilon y^{2}} \mathrm{~d} y
$$

and this is bounded in $\varepsilon=t / x^{2}>0$. So we arrive at

$$
\begin{gathered}
\int_{0}^{\infty} \mathrm{e}^{-t u} \frac{\operatorname{Bi}(-u) \operatorname{Ai}(-u-x)-\operatorname{Ai}(-u) \operatorname{Bi}(-u-x)}{\pi\left(\mathrm{Ai}^{2}(-u)+\mathrm{Bi}^{2}(-u)\right)} \mathrm{d} u \\
=\frac{x}{2 \pi t}\left(\frac{\pi}{t}\right)^{1 / 2} \mathrm{e}^{-x^{2} / 4 t}\left(1+\frac{1}{2} x t\right)+O(1) .
\end{gathered}
$$

Here $0<x \leq D$, and there is no particular constraint on $t$ other than just being positive. This completes the proof of Theorem 2.1 .

\section{Proof of Theorem 2.2}

We first study the contribution of the negative integration range $u \leq 0$ in (2.1). We have, for any $D>0$,

$$
\begin{aligned}
\int_{0}^{\infty} & \mathrm{e}^{-t u} \frac{\operatorname{Bi}(-u) \operatorname{Ai}(-u-x)-\operatorname{Ai}(-u) \operatorname{Bi}(-u-x)}{\pi\left(\operatorname{Ai}^{2}(-u)+\mathrm{Bi}^{2}(-u)\right)} \mathrm{d} u \\
& =\frac{\operatorname{Bi}(0) \operatorname{Ai}(-x)-\operatorname{Ai}(0) \operatorname{Bi}(-x)}{\pi\left(\operatorname{Ai}^{2}(0)+\mathrm{Bi}^{2}(0)\right)} \frac{1}{t}+O\left(t^{-2}\right), \quad 0 \leq x \leq D, t \geq 1 .
\end{aligned}
$$

This follows easily from the smoothness and uniform boundedness of

$$
u \geq 0 \mapsto \frac{\operatorname{Bi}(-u) \operatorname{Ai}(-u-x)-\operatorname{Ai}(-u) \operatorname{Bi}(-u-x)}{\pi\left(\mathrm{Ai}^{2}(-u)+\mathrm{Bi}^{2}(-u)\right)},
$$

and its derivative when $x$ is bounded.

Let us next study the contribution of the positive integration range $u \geq 0$ in (2.1). We have, from (5.1)-(5.2),

$$
\mathrm{Ai}^{2}(u)+\mathrm{Bi}^{2}(u)=\frac{\exp \left(4 u^{3 / 2} / 3\right)}{\pi \sqrt{u}}\left(1+O\left(u^{-3 / 2}\right)\right), \quad u>0 .
$$

Furthermore, for $u>2 x$,

$$
\begin{aligned}
& \operatorname{Ai}(u-x) \operatorname{Bi}(u)-\operatorname{Ai}(u) \operatorname{Bi}(u-x) \\
& =\frac{1}{2 \pi u^{1 / 4}(u-x)^{1 / 4}}\left[\exp \left(\frac{2}{3} u^{3 / 2}-\frac{2}{3}(u-x)^{3 / 2}\right)\left(1+O\left(u^{-3 / 2}\right)\right)\right. \\
& \left.\quad-\exp \left(-\frac{2}{3} u^{3 / 2}+\frac{2}{3}(u-x)^{3 / 2}\right)\left(1+O\left(u^{-3 / 2}\right)\right)\right] .
\end{aligned}
$$


Using (5.5), with $-x$ instead of $x$, we approximate, for $u>2 x, x \leq D$,

$$
\begin{aligned}
& \mathrm{e}^{t u} \frac{\operatorname{Bi}(u) \operatorname{Ai}(u-x)-\operatorname{Ai}(u) \operatorname{Bi}(u-x)}{\pi\left(\operatorname{Ai}^{2}(u)+\operatorname{Bi}^{2}(u)\right)} \\
& =\frac{1}{2 \pi}\left(\frac{u}{u-x}\right)^{1 / 4} \mathrm{e}^{t u-4 u^{3 / 2} / 3}\left(\mathrm{e}^{x \sqrt{u}-x^{2} / 4 \sqrt{u}}\left(1+O\left(u^{-3 / 2}\right)\right)\right. \\
& \left.-\mathrm{e}^{-x \sqrt{u}+x^{2} / 4 \sqrt{u}}\left(1+O\left(u^{-3 / 2}\right)\right)\right) .
\end{aligned}
$$

The factor $\exp \left(t u-\frac{4}{3} u^{3 / 2}\right)$ is maximal $\frac{1}{12} t^{3}$ at $u=\frac{1}{4} t^{2}$. Since $(\mathrm{d} / \mathrm{d} u)^{2}\left[t u-\frac{4}{3} u^{3 / 2}\right]=2 / t$ at $u=\frac{1}{4} t^{2}$, the contribution from the set $\left|u-\frac{1}{4} t^{2}\right| \geq t$ is exponentially small in $t \geq 1$. On the range $\left|u-\frac{1}{4} t^{2}\right| \leq t$ there is the approximation

$$
\begin{aligned}
& \mathrm{e}^{t u} \frac{\operatorname{Bi}(u) \operatorname{Ai}(u-x)-\operatorname{Ai}(u) \operatorname{Bi}(u-x)}{\pi\left(\operatorname{Ai}^{2}(u)+\operatorname{Bi}^{2}(u)\right)} \\
& \quad=\frac{1}{2 \pi} \mathrm{e}^{t u-4 u^{3 / 2} / 3}\left(\mathrm{e}^{x \sqrt{u}-x^{2} / 2 t}\left(1+O\left(t^{-2}\right)\right)-\mathrm{e}^{-x \sqrt{u}+x^{2} / 2 t}\left(1+O\left(t^{-2}\right)\right)\right) .
\end{aligned}
$$

We hence aim at an asymptotic analysis of the integrals

$$
J( \pm x, t)=\int_{0}^{\infty} \mathrm{e}^{t u-4 u^{3 / 2} / 3 \pm x \sqrt{u}} \mathrm{~d} u
$$

Here we follow the treatment of De Bruijn [5, Section 4.5] for the asymptotics of the gamma function from its standard integral representation. In particular, the various substitutions below are motivated by what is done there.

With the substitution $u=t^{2}(1+y)^{2} / 4$, with $y \geq-1$, we obtain

$$
J( \pm x, t)=\frac{1}{2} t^{2} \mathrm{e}^{t^{3} / 12 \pm x t / 2} \int_{-1}^{\infty}(1+y) \mathrm{e}^{ \pm x t y / 2} \mathrm{e}^{-t^{3}\left(y^{2}+2 y^{3} / 3\right) / 4} \mathrm{~d} y .
$$

Consider the mapping

$$
y \in[-1, \infty) \mapsto \varphi(y)=y^{2}+\frac{2}{3} y^{3} .
$$

This $\varphi$ decreases strictly on $[-1,0]$ and increases strictly on $[0, \infty)$, and we have $\varphi^{\prime}(-1)=$ $\varphi^{\prime}(0)=0, \varphi(-1)=\frac{1}{3}$, and $\varphi(0)=0$. We make the substitution

$$
y^{2}+\frac{2}{3} y^{3}=z^{2}
$$

where $z \in(-1 / \sqrt{3}, \infty)$, and where we require $z$ and $y=y(z)$ to have the same sign. Thus, we have

$$
y(z)\left(1+\frac{2}{3} y(z)\right)^{1 / 2}=z, \quad(1+y(z)) y^{\prime}(z)=\frac{z}{y(z)}=\left(1+\frac{2}{3} y(z)\right)^{1 / 2},
$$

and it follows that

$$
J( \pm x, t)=\frac{1}{2} t^{2} \mathrm{e}^{t^{3} / 12 \pm x t / 2} \int_{-1 / \sqrt{3}}^{\infty}\left(1+\frac{2}{3} y(z)\right)^{1 / 2} \mathrm{e}^{ \pm x t y(z) / 2} \mathrm{e}^{-t^{3} z^{2} / 4} \mathrm{~d} z .
$$


We then use the final substitution $w=t z$ to obtain

$$
\begin{aligned}
\int_{-1 / \sqrt{3}}^{\infty} & \left(1+\frac{2}{3} y(z)\right)^{1 / 2} \mathrm{e}^{ \pm x t y(z) / 2} \mathrm{e}^{-t^{3} z^{2} / 4} \mathrm{~d} z \\
= & \frac{1}{t} \int_{-t / \sqrt{3}}^{\infty}\left(1+\frac{2}{3} y\left(\frac{w}{t}\right)\right)^{1 / 2} \mathrm{e}^{ \pm x t y(w / t) / 2} \mathrm{e}^{-t w^{2} / 4} \mathrm{~d} w
\end{aligned}
$$

The integration range of the latter integral can be limited to $w \in[-1,1]$ at the expense of an exponentially small error in $t \geq 1$. On this range, we have

$$
y\left(\frac{w}{t}\right)=\frac{w}{t}-\frac{w^{2}}{3 t^{2}}+\frac{5 w^{3}}{18 t^{3}}-\frac{8 w^{4}}{27 t^{4}}+\frac{385 w^{5}}{1080 t^{5}}-\cdots,
$$

where we have used the Bürmann-Lagrange inversion theorem,

$$
\begin{aligned}
y(z) & =\sum_{k=1}^{\infty} \frac{1}{k !}\left(\frac{\mathrm{d}}{\mathrm{d} y}\right)^{k-1}\left[\left(1+\frac{2}{3} y\right)^{-k / 2}\right] \text { (0) } z^{k} \\
& =z \sum_{k=0}^{\infty} \frac{\Gamma(3 k / 2+1 / 2)}{\Gamma(k / 2+1 / 2)} \frac{(-2 z / 3)^{k}}{(k+1) !}, \quad|z| \leq \frac{1}{\sqrt{3}},
\end{aligned}
$$

to solve $y(z)=y$ from (6.3). Thus, with an exponentially small error in $t \geq 1$, we have

$$
\begin{aligned}
& \int_{-t / \sqrt{3}}^{\infty}\left(1+\frac{2}{3} y\left(\frac{w}{t}\right)\right)^{1 / 2} \mathrm{e}^{ \pm x t y(w / t) / 2} \mathrm{e}^{-t w^{2} / 4} \mathrm{~d} w \\
& \quad=\int_{-1}^{1}\left\{\text { even part of }\left[\left(1+\frac{2}{3} y\left(\frac{w}{t}\right)\right)^{1 / 2} \mathrm{e}^{ \pm x t y(w / t) / 2}\right]\right\} \mathrm{e}^{-t w^{2} / 4} \mathrm{~d} w
\end{aligned}
$$

It is an easy, though somewhat laborious task, to show that

$$
\begin{aligned}
& \text { even part of }\left[\left(1+\frac{2}{3} y\left(\frac{w}{t}\right)\right)^{1 / 2} \mathrm{e}^{ \pm x t y(w / t) / 2}\right] \\
& =1+\frac{1}{8} x^{2} w^{2}+O\left(w^{4}+\frac{w^{2}}{t}+t^{-2}\right), \quad|w| \leq 1,0 \leq x \leq D .
\end{aligned}
$$

From

$$
\int_{-\infty}^{\infty} w^{2 j} \mathrm{e}^{-t w^{2} / 4} \mathrm{~d} w=\frac{2 \sqrt{\pi}}{\sqrt{t}}(2 j-1) ! !\left(\frac{2}{t}\right)^{j},
$$

it is then found that, with an exponentially small error in $t \geq 1$,

$$
J( \pm x, t)=\sqrt{\pi t} \mathrm{e}^{t^{3} / 12 \pm x t / 2}\left(1+\frac{x^{2}}{4 t}+O\left(t^{-2}\right)\right) .
$$

We thus find from (6.2) and (6.4) that, when $x$ is bounded and $t \geq 1$,

$$
\begin{aligned}
\int_{0}^{\infty} & \mathrm{e}^{t u} \frac{\operatorname{Bi}(u) \operatorname{Ai}(u-x)-\operatorname{Ai}(u) \operatorname{Bi}(u-x)}{\pi\left(\operatorname{Ai}^{2}(u)+\operatorname{Bi}^{2}(u)\right)} \mathrm{d} u \\
& =\frac{1}{2 \pi}\left(\mathrm{e}^{-x^{2} / 2 t} J(x, t)\left(1+O\left(t^{-2}\right)\right)-\mathrm{e}^{x^{2} / 2 t} J(-x, t)\left(1+O\left(t^{-2}\right)\right)\right)
\end{aligned}
$$




$$
\begin{aligned}
& =\frac{t^{1 / 2}}{\sqrt{\pi}} \mathrm{e}^{t^{3} / 12}\left(\sinh \left(\frac{1}{2} x t-\frac{x^{2}}{4 t}\right)+\cosh \left(\frac{1}{2} x t-\frac{x^{2}}{4 t}\right) O\left(t^{-2}\right)\right) \\
& =\frac{t^{1 / 2}}{\sqrt{\pi}} \mathrm{e}^{t^{3} / 12} \sinh \left(\frac{1}{2} x t\right)\left(1+O\left(t^{-1}\right)\right), \quad t \geq 1,
\end{aligned}
$$

uniformly in $x \in[\varepsilon, D]$ for any $\varepsilon>0$. The contribution of the integration range $u \leq 0$ is $O\left(t^{-1}\right)$ and, thus, is exponentially small compared to the contribution of the integration range $u \geq 0$ (see (6.5)). From this, the result follows.

We finally show statements $(2.5)$ and $(2.6)$ concerning $\mathcal{F}_{3}(t)$. The stationary points of $\mathcal{F}_{3}(t)$ are the two roots

$$
\frac{4}{3} a \pm \frac{2}{3}\left(a^{2}+3 b\right)^{1 / 2}
$$

of the equation

$$
(t-a)^{2}=\frac{1}{4} t^{2}+b
$$

with a relative minimum for $\mathcal{F}_{3}(t)$ when the ' + ' sign is taken in (6.6). Using

$$
t_{2}=\frac{4}{3} a+\frac{2}{3}\left(a^{2}+3 b\right)^{1 / 2}=2 a+\frac{b}{a}-\frac{3}{4} \frac{b^{2}}{a^{3}}+O\left(\frac{b^{3}}{a^{5}}\right),
$$

together with (6.7) for $t=t_{2}$, we compute $\mathcal{F}_{3}\left(t_{2}\right)$ as

$$
\begin{aligned}
\mathscr{F}_{3}\left(t_{2}\right) & =-\frac{1}{4} a t_{2}^{2}-2 b t_{2}+5 a b+a^{3} \\
& =-\frac{1}{4} a\left(2 a+\frac{b}{a}-\frac{3}{4} \frac{b^{2}}{a^{3}}+O\left(\frac{b^{3}}{a^{5}}\right)\right)^{2}-2 b\left(2 a+\frac{b}{a}+O\left(\frac{b^{2}}{a^{3}}\right)\right)+5 a b+a^{3} \\
& =-\frac{3}{2} \frac{b^{2}}{a}+O\left(\frac{b^{3}}{a^{3}}\right) .
\end{aligned}
$$

Also,

$$
\mathcal{F}_{3}^{\prime \prime}\left(t_{2}\right)=\frac{9}{2} t_{2}-6 a=3 a+O\left(\frac{b}{a}\right) .
$$

\section{Acknowledgements}

The authors thank Svante Janson for his careful reading of an earlier version of the manuscript. The work of RvdH was supported in part by the Netherlands Organisation for Scientific Research (NWO).

\section{References}

[1] Abramowitz, M. and Stegun, I. A. (eds) (1984). Handbook of Mathematical Functions with Formulas, Graphs, and Mathematical Tables. John Wiley, New York.

[2] Aldous, D. (1997). Brownian excursions, critical random graphs and the multiplicative coalescent. Ann. Prob. 25, 812-854.

[3] Bhamidi, S., van der Hofstad, R. and van Leeuwaarden, J. S. H. (2009). Scaling limits for critical inhomogeneous random graphs with finite third moments. Preprint. Available at http://arxiv.org/abs/0907.4279v2.

[4] Bollobás, B. (2001). Random Graphs (Camb. Stud. Adv. Math. 73), 2nd edn. Cambridge University Press.

[5] De Bruijn, N. G. (1981). Asymptotic Methods in Analysis, 3rd edn. Dover, New York.

[6] Doney, R. A. And Yor, M. (1998). On a formula of Takács for Brownian motion with drift. J. Appl. Prob. 35, 272-280.

[7] Gordillo, L. F., Marion, S. A., Martin-Löf, A. and Greenwood, P. E. (2008). Bimodal epidemic size distributions for near-critical SIR with vaccination. Bull. Math. Biol. 70, 589-602. 
[8] Groeneboom, P. (1989). Brownian motion with a parabolic drift and Airy functions. Prob. Theory Relat. Fields 81, 79-109.

[9] JANSON, S. (2007). Brownian excursion area, Wright's constants in graph enumeration, and other Brownian areas. Prob. Surveys 4, 80-145.

[10] JAnson, S. AND SPEnCER, J. (2007). A point process describing the component sizes in the critical window of the random graph evolution. Combinatorics Prob. Comput. 16, 631-658.

[11] Janson, S., ŁuCZaK, T. And Rucinski, A. (2000). Random Graphs. Wiley-Interscience, New York.

[12] Lalley, S. P. (2007). Critical scaling of stochastic epidemic models. In Asymptotics: Particles, Processes and Inverse Problems (IMS Lecture Notes Monogr. Ser. 55), Institute for Mathematical Statistics, Beachwood, OH, pp. 167-178.

[13] Łuczak, T., Pittel, B. And Wierman, J. C. (1994). The structure of a random graph at the point of the phase transition. Trans. Amer. Math. Soc. 341, 721-748.

[14] Martin-LöF, A. (1986). Symmetric sampling procedures, general epidemic processes and their threshold limit theorems. J. Appl. Prob. 23, 265-282.

[15] Martin-LöF, A. (1998). The final size of a nearly critical epidemic, and the first passage time of a Wiener process to a parabolic barrier. J. Appl. Prob. 35, 671-682.

[16] Nachmias, A. and Peres, Y. (2007). The critical random graph, with martingales. Preprint. Available at http://arxiv.org/abs/math/0512201v4.

[17] Pittel, B. (2001). On the largest component of the random graph at a nearcritical stage. J. Combinatorial Theory B 82, 237-269.

[18] Startsev, A. N. (2001). Asymptotic analysis of the general stochastic epidemic with variable infectious periods. J. Appl. Prob. 38, 18-35.

[19] Takács, L. (1989). Ballots, queues and random graphs. J. Appl. Prob. 26, 103-112.

[20] Turova, T. S. (2009). Diffusion approximation for the components in critical inhomogeneous random graphs of rank 1. Preprint. Available at http://arxiv.org/abs/0907.0897v2.

[21] VAn der Hofstad, R., Kager, W. ANd Müller, T. (2009). A local limit theorem for the critical random graph. Electron. Commun. Prob. 14, 122-131. 